\title{
Sleep-disordered Breathing in Heart Failure: A Complex Bidirectional Pathophysiology
}

\author{
Sumita Agrawal ${ }^{1}$, Nitesh Gupta ${ }^{2}$, Pranav Ish $^{3}$, Shibdas Chakrabarti ${ }^{4}$
}

\begin{abstract}
Heart failure (HF) shares a bidirectional cause and effect relationship with sleep-disordered breathing (SDB). This review aims to address the epidemiology, etiopathogenesis, and treatment for such patients as early suspicion, and treatment is the key to improved morbidity and mortality. Keywords: Central sleep apnea, Heart failure, Obstructive sleep apnea, Sleep-disordered breathing. Indian Journal of Sleep Medicine (2019): 10.5005/jp-journals-10069-0045
\end{abstract}

\section{INTRODUCTION}

Heart failure (HF) is a clinical entity characterized by typical symptoms (e.g., breathlessness, ankle swelling, and fatigue) with/ without signs (e.g., elevated jugular venous pressure, crackles, and peripheral edema). It is affected by a structural or functional cardiac abnormality, ending in a reduced cardiac output with or without increased intracardiac pressures at rest or during stress. ${ }^{1}$ The aging of population and prolongation of the lives of cardiac patients by modern therapies have led to an increment in the prevalence of HF. Besides, we have also seen an increase in sleep-disordered breathing (SDB) of varying patterns in these patients. The mortality rate in patients suffering from SDB-HF is unacceptably high, making early detection of this condition imperative. ${ }^{2}$

\section{Heart Failure}

The existing definition of HF restricts itself to stages at which clinical symptoms manifest. Before clinical symptoms manifest, patients can exhibit asymptomatic structural or functional cardiac abnormalities [systolic or diastolic left ventricular (LV) dysfunction], which are precursors of HF. Heart failure encompasses a wide range of patients, of those with normal left ventricular ejection fraction (LVEF) [typically considered as $\geq 50 \%$; HF with preserved ejection fraction (HFpEF)] to those with reduced LVEF [typically considered as $40 \%$; HF with reduced ejection fraction (HFrEF)]. Patients with an LVEF in the range of $40-49 \%$ denote a "gray area," which we now define as HFmrEF. ${ }^{1}$ An unusual electrocardiogram and/or plasma levels of B-type natriuretic peptide (BNP) $>35 \mathrm{pg} / \mathrm{mL}$ and/ or N-terminal pro-BNP (NT-proBNP) $>125 \mathrm{pg} / \mathrm{mL}$ secure a diagnosis of HFpEF, HFmrEF, or HFrEF more likely. The subsequent step comprises an advanced workup in case of initial evidence of HFpEF/ HFmrEF. It comprises an objective demonstration of structural and/or functional alterations of the heart as the underlying cause for the clinical presentation. Differentiation of patients with HF based on LVEF is important due to diverse underlying etiology, demographics, comorbidities, and response to therapies. ${ }^{3}$

\section{Heart Failure: Epidemiology and ETIOPATHOGENESIS}

The prevalence of HF depends on the definition implemented, 1-2\% of the adult population in developed nations, rising to $\geq 10 \%$ among
${ }^{1}$ Department of Pulmonary Medicine, Medipulse Hospital, Jodhpur, Rajasthan, India

${ }^{2-4}$ Department of Pulmonary, Critical Care and Sleep Medicine, Vardhman Mahavir Medical College and Safdarjung Hospital, New Delhi, India

Corresponding Author: Nitesh Gupta, Department of Pulmonary, Critical Care and Sleep Medicine, Vardhman Mahavir Medical College and Safdarjung Hospital, New Delhi, India, Phone:+91 9873096364, e-mail: niteshgupta2107@gmail.com

How to cite this article: Agrawal S, Gupta N, Ish P, et al. Sleep-disordered Breathing in Heart Failure: A Complex Bidirectional Pathophysiology. Indian J Sleep Med 2019;14(4):70-75.

Source of support: Nil

Conflict of interest: None

people $>70$ years of age. ${ }^{4}$ Among HF population, the prevalence of $\mathrm{HFpEF}$ ranges from 22 to $73 \%$, depending on the definition used, the clinical setting (primary care, hospital clinic, and hospital admission), age and sex of the examined population, and previous myocardial infarction (MI). ${ }^{1}$

The etiology of HF is diverse; patients will have several different pathologies - cardiovascular and noncardiovascular-that conspire to cause HF. Identification of these different pathologies should be part of the diagnostic workup (Table 1). In the 1970s, in the United States and Europe, hypertension and coronary disease, particularly $\mathrm{MI}$, were the essential causes of HF. ${ }^{5,6}$ However, recently, coronary disease and diabetes mellitus have become frequently responsible for $\mathrm{HF}$, while hypertension and valve disease have become less prevalent because of advances in detection and therapy. ${ }^{7}$

In a 19-year follow-up study of 13,643 men and women, National Health and Nutrition Examination Survey (NHANES I), the risk determinants for HF were coronary heart disease, cigarette smoking, hypertension, obesity, diabetes mellitus, and valvular heart disease. However, the valvular disease is a frequently probable cause of HF at older ages, with calcific aortic stenosis being the most common disorder requiring surgery. ${ }^{7}$

The predisposing conditions commonly associated with HFpEF and HFrEF include older age, hypertension, coronary disease, and diabetes mellitus. Patients with HFpEF tend to be older, more frequently have hypertension, are overweight, and are more often women, compared with patients with HFrEF. ${ }^{8}$ The PREVEND 
community-based cohort study of middle-aged subjects identified predictors of these two types of HF. In a multivariate analysis, incident HF was associated with the factors listed above as well as obesity, NT-proBNP, and highly sensitive troponin T (hs-TnT) in all subjects. In a comparison of etiology for HFpEF vs HFrEF, female sex, atrial fibrillation, increased urinary albumin excretion, and raised cystatin-C levels were preferentially associated with HFpEF. The male sex, smoking, hs-TnT, and prior MI were preferentially associated with HFrEF. ${ }^{9}$

\section{Sleep-disordered Breathing}

Sleep-disordered breathing defines disorders of plural chronic conditions in which partial or complete cessation of breathing occurs primarily and often exclusively during sleep. The characteristic feature of SDB is cycles of notable pauses in breathing with consequent hypoxia and incomplete neurological arousals that interrupt the architecture of sleep. The classification of SDB includes obstructive sleep apnea (OSA) and central sleep apnea (CSA). The apnea hypopnea index (AHI) grades the severity

Table 1: Predisposing conditions for heart failure

HF-predisposing conditions
Hypertension
Coronary artery disease
Valvular heart disease
Cigarette smoking
Diabetes mellitus
Overweight
Atrial fibrillation
Sleep apnea
Advancing age

as mild (6-14 episodes/hour), moderate, ${ }^{10-23}$ or severe $(>30)$ (Figs 1 and 2). ${ }^{24}$

Obstructive sleep apnea results from the absolute collapse of the pharynx and upper airways, whereas CSA arises from the variations in the central respiratory drive. During OSA, the abdominal and thoracic efforts increase to produce airflow. By contrast, respiratory movements are absent in CSA. ${ }^{25}$ Obstructive sleep apnea and CSA might stem from a common origin, and the patient can present with predominantly obstructive apneas at the beginning of the night that transforms into essentially central apneas toward the morning. ${ }^{26}$ The third form of SDB prevalent in patients with HF is mixed sleep apnea, designated by an initial central apnea event followed by an obstructive component.

\section{Sleep-disordered Breathing: Epidemiology AND ETIOPATHOgenesis}

The prevalence of OSA in otherwise healthy adults varies widely in the literature. It is $2-9 \%$ in women and $4-26 \%$ in men depending on the models used for the diagnosis but with a marked upsurge in prevalence in a previous couple of decades. ${ }^{27,28}$ The prevalence of CSA/Cheyne-Stokes respiration (CSR) in healthy adults seems to be below that of OSA, but no strong evidence has been presented on this issue so far. Age, sex, sleep stage, and several medical conditions reported affecting susceptibility to CSA. Breathing instability anticipated to occur at sleep onset when patients can oscillate between wakefulness, and various sleep stages are also a potential trigger for CSA. ${ }^{26}$

\section{Sleep-disordered Breathing in Heart FAILURE}

In the HF population with reduced LVEF ( $\leq 40 \%$, HFrEF), SDB is $50-75 \%$ of cases; however, SDB prevalence estimates in the HFpEF

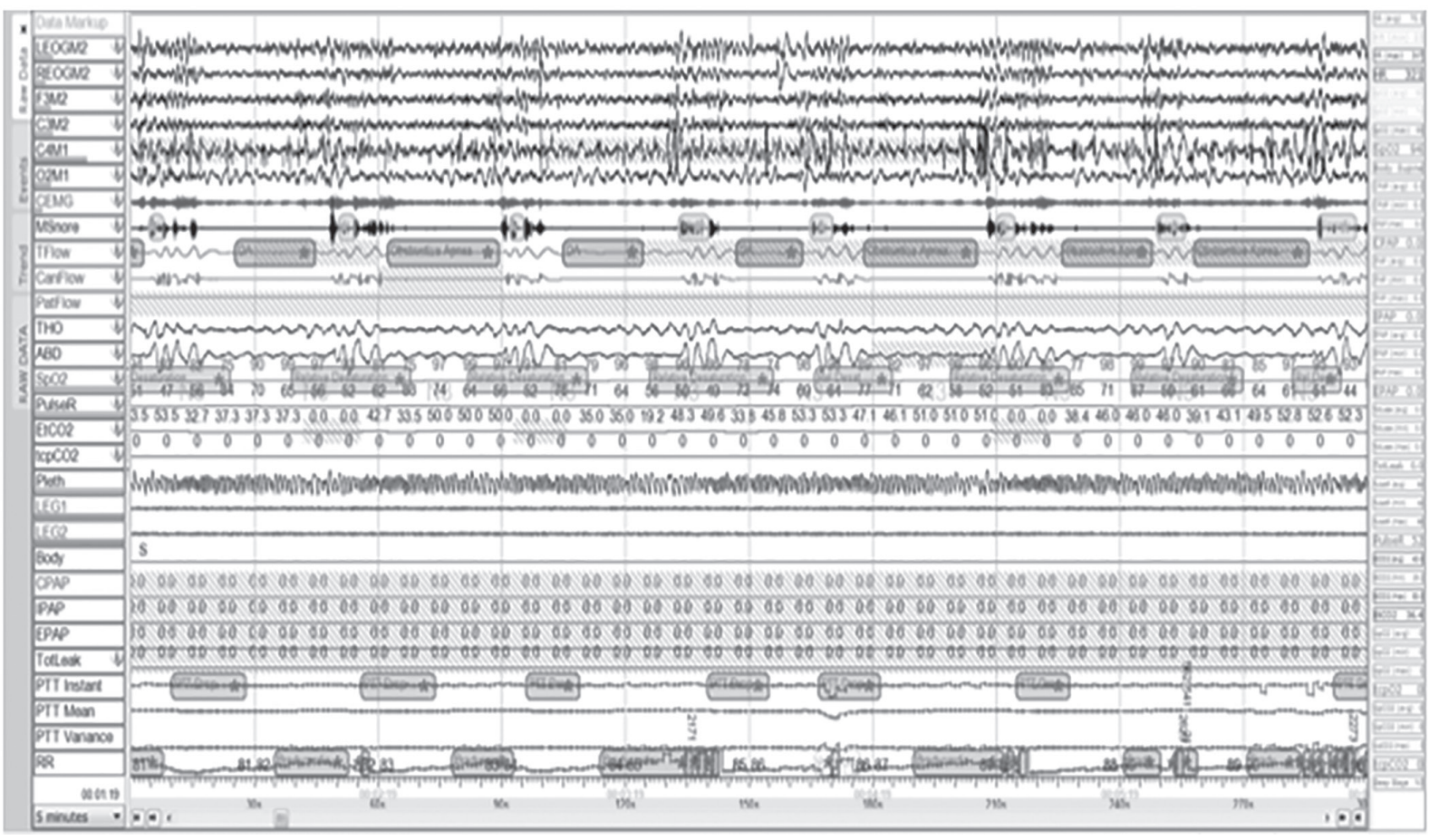

Fig. 1: A 5-minute epoch showing numerous obstructive sleep apneas associated with fall in nasal flow with preserved thoracic and abdominal efforts 


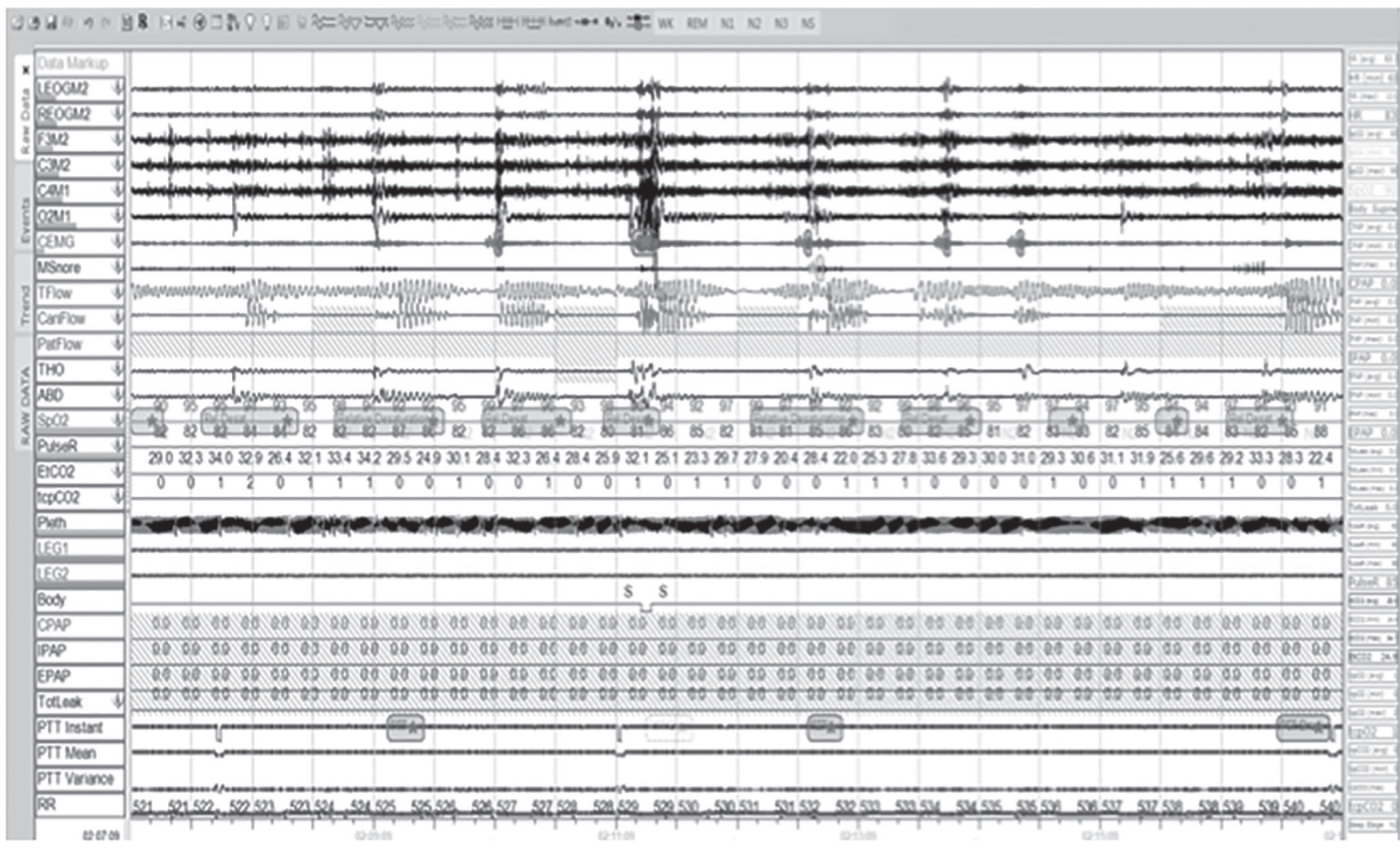

Fig. 2: A 5-minute epoch showing numerous central sleep apneas associated with fall in nasal flow with absent thoracic and abdominal efforts. The crescendo and decrescendo flow is suggestive of Cheyne-Stokes breathing

population is slightly unclear. The data about the prevalence of SDB in HFpEF are relatively scarce; existing studies support a prevalence of SDB of approximately $55 \% .^{10,11}$

The cross-sectional studies reported OSA prevalence (11-37\%), the prevalence being greater in men (38\%) than in women (31\%). ${ }^{12}$ The prospective sleep heart health study comprising 6,424 men and women indicated that the presence of OSA (defined as an $\mathrm{AHI} \geq 10 /$ hour) favored the appearance of HF independent of other known risk factors. ${ }^{13}$ In cross-sectional studies on SDB in patients with HF with LV systolic dysfunction, the frequency of CSA/CSR ranges between $28 \%$ and $82 \% .{ }^{10,12}$ This enormous range might depend on numerous variables, including the etiology and severity of HF. The principal risk factors for CSA/CSR in HF patients identified are male sex, advanced age, hypocapnia, atrial fibrillation, or presence of a pacemaker, except obesity. ${ }^{12,14}$ Interestingly, women with systolic dysfunction infrequently develop CSA/CSR.

\section{Pathogenesis of Obstructive Sleep Apnea in Heart Failure}

The collapse of the upper airways produces OSA during sleep in patients with anatomically narrowed and extremely compliant airways (Flowchart 1). The dysfunction of the motor neurons, liable for controlling the musculature within the pharynx, is accountable for collapse. In patients with HF, additional factors advocate the collapse of the upper airways. Marked oscillations in the ventilatory drive, resulting in periodic breathing, influence the upper airway narrowing. Also, the fluid shift in the supine position from the legs to central structures contributes to airway narrowing. ${ }^{15,16}$ Heart failure worsens the presence and severity of OSA, and OSA influences the progression to chronic HF through multiple mechanisms. Inspiration against a closed airway reduces intrathoracic pressure, increasing venous return to the right ventricle. The increase in preload, as mentioned earlier, causes a leftward shift of the interventricular septum that further reduces the LV function. The negative intrathoracic pressure is also liable for an increased LV transmural pressure, which increases the afterload, further impairing the function of an already failing left ventricle. ${ }^{17}$ These recurrent events that accompany repeated obstructive apnea cause a further increase in the already elevated sympathetic activity in patients with HF, documented by increased plasma catecholamine. The association of rehashed cycles of apneas and hypopneas induces endothelial dysfunction. Also noted is increased plasma concentration of inflammatory markers, increased platelet aggregability, and increased variability in blood pressure and heart rate. ${ }^{18}$

\section{Pathogenesis of Central Sleep Apnea in Heart Failure}

The arterial partial pressure of $\mathrm{CO}_{2}\left(\mathrm{PaCO}_{2}\right)$ is the most critical factor controlling ventilatory drive during both wake and sleep. Periodic breathing is defined by central apnea, which transpires when the $\mathrm{PaCO}_{2}$ drops below the apneic threshold, followed by a hyperventilation phase. ${ }^{18}$ Whenever the chemical drive of breathing prevails over the cortical influence on the respiratory controller-as typically transpires during sleep-the patient becomes apneic until the $\mathrm{PaCO}_{2}$ increases above the apnea threshold, followed by a decline in the arterial partial pressure of $\mathrm{O}_{2}\left(\mathrm{PaO}_{2}\right)$. The alternating pattern of apnea and hyperpnea sustains because of the continuing oscillations of $\mathrm{PaCO}_{2}$ around the apnea threshold, associated with synchronous oscillations in $\mathrm{PaO}_{2}$. In LV failure patients, increased pulmonary venous pressure leads to pulmonary congestion, thereby stimulating pulmonary stretch receptors, hyperventilation, and hypocapnia. ${ }^{19}$ The stimulation of stretch receptors also increases the respiratory center sensitivity to $\mathrm{CO}_{2}$ through their vagal afferents. 
Flowchart 1: Pathogenesis of sleep-disordered breathing in heart failure

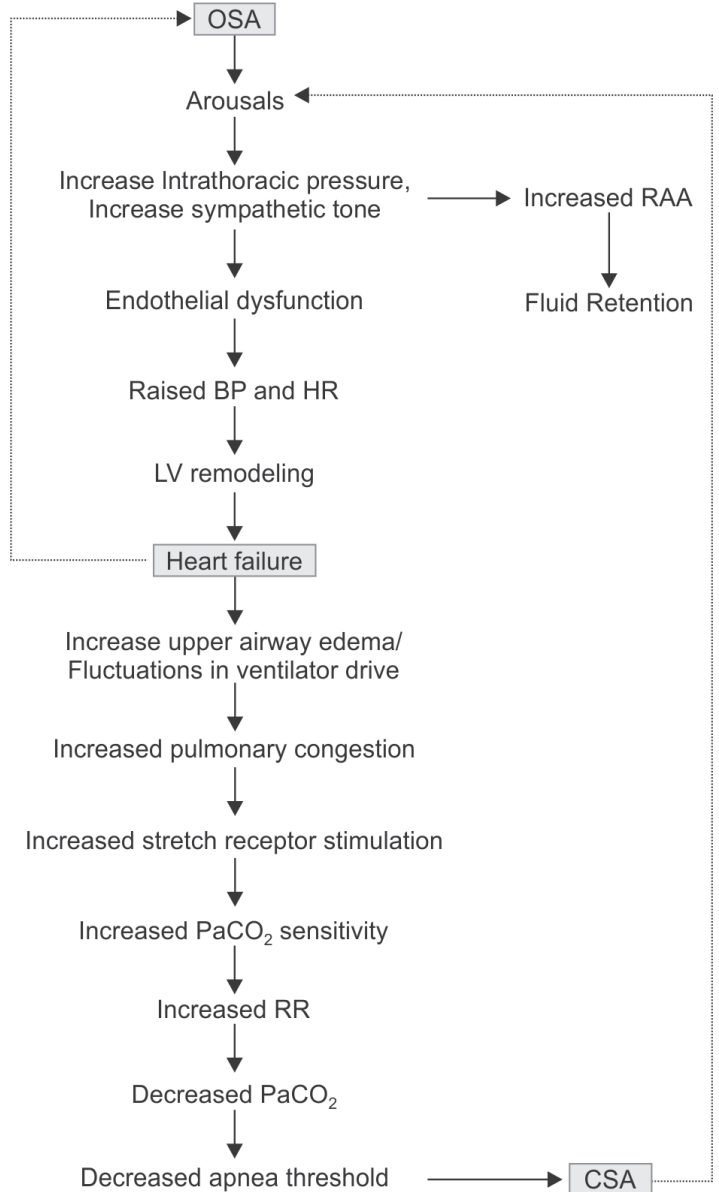

OSA, obstructive sleep apnea; RAA, renin-angiotensin aldosterone; HR, heart failure; LV, left ventricle; RR pulse rate; CSA, central sleep apnea

When patients with HF and peripheral edema lie supine, increased venous return from the extremities causes a rostral fluid shift, and pulmonary congestion further stimulates vagal receptors in the lungs to elicit reflex hyperventilation. ${ }^{20}$ Indeed, central apneas are more prevalent in the supine position, and sleeping on one side can lead to the amelioration of the severity of CSA and CSR. ${ }^{21}$

Overall, CSA/CSR has been recognized more as a consequence than a cause of HF. However, HF and CSA patients are characterized by a high sympathetic activation during both day and night. This sympathetic activation is linked to the frequency of apneas. Additionally, the frequency and severity of hypoxia through chemoreflex activation contribute to the degree of sympathetic activation. These mechanisms suggest that CSA can have a causative role in worsening the clinical condition of patients with HF, emphasizing the existence of a bidirectional relationship between two conditions. The bidirectional relationship has potential therapeutic implications, given the evidence that the treatment of CSA can diminish sympathetic activity. ${ }^{22}$

\section{Sleep Quality in Heart Failure}

Patients with HF frequently complain of sleep fragmentation or non-restorative sleep, challenges in initiating sleep, or waking up too early in the morning. Epidemiological data display a higher prevalence of chronic insomnia in patients with $\mathrm{HF}$ than the general population (33\% vs $10-15 \%) .{ }^{23}$ In distinct cases, insomnia can be secondary. This is probably caused by HF itself or by adverse effects of commonly prescribed HF medications, such as angiotensinreceptor blockers, loop diuretics, and $\beta$-blockers. Nocturia and orthopnea are commonly reported by patients with HF and are additional reasons of poor sleep quality. ${ }^{29}$

Oddly, HF patients with OSA or CSA do not complain of daytime sleepiness, snoring, or choking presumably because of the high sympathetic tone in $\mathrm{HF}^{30}$ The Canadian Cardiovascular Society recommends considering OSA in HF patients manifesting as paroxysmal or recurrent atrial fibrillation, refractory hypertension, raised body mass index, and unanticipated pulmonary hypertension or right ventricular dysfunction. Furthermore, SDB should also be considered in HF patients with malignant ventricular arrhythmias particularly at night. ${ }^{31}$

\section{Diagnosis of Sleep-disordered Breathing in Heart Failure}

The sleep apnea diagnosis in patients with HF requires nocturnal monitoring of specific signals to recognize the peculiar mechanical characteristics of sleep breathing typical of OSA and CSA and to quantify the AHI. A formal diagnosis of OSA is made in instances when $\mathrm{AHI}>15 /$ hour or when $\mathrm{AHI}>5$ /hour with concomitant presence of at least one of the following symptoms: sleepiness; non-restorative sleep; fatigue or insomnia; waking up with breath-holding, gasping, or choking; habitual snoring, breathing interruptions, or both recorded by a bed partner or observer; hypertension; mood disorder; cognitive dysfunction; coronary artery disease; stroke; congestive HF; atrial fibrillation; or type II diabetes mellitus. Furthermore, diagnosis of CSA requires a component $\mathrm{AHI}$ of five or more with or without central hypopneas per hour of sleep, with additional criteria for specific subtypes of CSA. ${ }^{24,32}$

The current standard diagnostic tool for SDB is overnight polysomnography. This requires the simultaneous monitoring of sleep electroencephalogram, eye movement, chin electromyogram, cardiac rhythm, body position, oxyhemoglobin saturation, oronasal flow, and detection of respiratory effort with noninvasive methods that can discriminate between obstructive and central events. The intraoesophageal pressure assessment as a measurement of respiratory effort is an alternative method, although less commonly performed. The assessment of the effect of SDB on cardiovascular function is done by measurement of the time required for pulse waves to reach peripheral arteries, defined as pulse transit time. Pulse transit time can also be used in the assessment of patients with $\mathrm{HF}$ and SDB, with better results when combined with capnography. ${ }^{33}$

Whether SDB occurs as a consequence of HFpEF or SDB induces, diastolic dysfunction is still a matter of debate. Fung et al. investigated 68 OSA patients for parameters of diastolic dysfunction; the results stated that more severe SDB was associated with a higher degree of diastolic dysfunction. ${ }^{34}$ These results were also supported by Otto et al., who compared 23 otherwise healthy patients with OSA with 18 patients without OSA and found an increased left atrial volume index as well as abnormal diastolic filling parameters in the OSA group. ${ }^{35}$ Besides an increased left atrial volume index, Romero-Corral et al. reported an association between SDB and an impaired myocardial performance index. ${ }^{36}$ Correspondingly, Sidana et al. found a higher prevalence of diastolic dysfunction in moderate-to-severe OSA than in those with mild or no OSA. ${ }^{37}$ The main reason for this could be repeated nocturnal hypoxemias leading to sympathetic nerve activation with a consequent increase in hormonal activation and arterial blood 
pressure, thus predisposing to wall thickening and compromised diastolic function.

In HFpEF, pulmonary congestion may arise from a compromised LV filling pattern, which supports the theory, that SDB, and CSA in particular, could be a consequence of HFpEF. Bitter et al. correlated pulmonary capillary wedge pressure (PCWP) and $\mathrm{AHI}$ in their entire cohort, and the CSA group specifically support this theory. ${ }^{38}$ Bucca et al. showed that the diuretic treatment of HFpEF produces a significant decrease in $\mathrm{AHI}$, possibly due to a reduction in pulmonary congestion. ${ }^{39}$

\section{TREATMENT}

Currently, the optimization of available HF therapies is still the gold standard for the management of patients with HF. Patients with even mild OSA and associated comorbidities need treatment for OSA. The first-line therapy for moderate-to-severe OSA is continuous positive airway pressure (CPAP). Oral appliance and upper airway surgery are other modalities of treatment. ${ }^{40}$ In the case of CSA/Cheyne-Stokes breathing (CSB) with HF apart from optimizing HF therapy, treatment options also include supplemental oxygen, CPAP, bi-level positive airway pressure (BPAP) with backup rate, adaptive servo-ventilation (ASV), or transplant. ${ }^{41}$

One randomized trial of CPAP provided preliminary evidence of prolonged survival in patients with CSB-CSA treated with CPAP. ${ }^{12}$ The Canadian continuous positive airway pressure trial subsequently investigated the ability of CPAP to improve mortality for patients with CSA and HF. This large, multicenter, randomized, controlled trial compared CPAP and standard treatment for patients with chronic HF and CSB-CSA. ${ }^{42}$ Unfortunately, the trial failed to show a survival benefit with CPAP treatment, and there was a trend for higher early mortality in the CPAP group. The outcomes of the study were disappointing, but the analysis showed that only about $50 \%$ of patients responded to CPAP (defined as an $\mathrm{AHI}<15$ /hour on a sleep study after 3 months of treatment). Several studies have documented that ASV is well tolerated and effective in CSB-CSA patients. The SERVE-HF trial had studied the impact of ASV in CSB-CSA patients in a randomized control design. The trial failed to demonstrate any mortality benefit. ${ }^{43}$ Patients in the ASV group had significantly higher mortality than the control group. A plausible explanation for the result is protective nature of CSA by resting of respiratory muscles, attenuation of excessive sympathetic nervous system activity, evasion of hypercapnic acidosis, hyperventilation-related increases in end-expiratory lung volume, and intrinsic positive airway pressure. Another feasible explanation could be the application of positive airway pressure may impair cardiac function in at least some patients with HF. The ongoing ADVENT-HF trial is designed to test whether ASV improves cardiovascular outcomes in HF patients with sleep apnea. The preliminary results show good compliance to therapy and no safety concerns as yet. ${ }^{44} \mathrm{~A}$ novel approach to the treatment of CSB-CSA is a stimulation of the phrenic nerve. A pulse generator is implanted to stimulate the diaphragm during sleep, aiming at maintaining regular respiratory excursions. ${ }^{45}$ Abraham et al. reported a $55 \%$ decrease in $\mathrm{AHI}$ at 3 months and a considerable improvement of sleep efficiency, oxygen saturation, and quality of life. ${ }^{46}$

Management of SDB in HF is complex and requires the combined role of cardiologist and pulmonologist. The screening of patients of HF for SDB should be routinely done, and suspected cases should undergo level I polysomnography.

\section{References}

1. Ponikowski P, Voors AA, Anker SD, et al. 2016 ESC guidelines for the diagnosis and treatment of acute and chronic heart failure. Eur Heart J 2016;37(27):2129-2200. DOI: 10.1093/eurheartj/ehw128.

2. Amir $\mathrm{O}$, Reisfeld $\mathrm{D}$, Sberro $\mathrm{H}$, et al. Implications of cheyne-stokes breathing in advanced systolic heart failure. Clin Cardiol 2010;33(3): 8-12. DOI: 10.1002/clc.20521.

3. Butler J, Fonarow GC, Zile MR, et al. Developing therapies for heart failure with preserved ejection fraction. JACC Hear Fail 2014;2(2): 97-112. DOI: 10.1016/j.jchf.2013.10.006.

4. Mosterd A, Hoes AW. Clinical epidemiology of heart failure. Heart 2007;93(9):1137-1146. DOI: 10.1136/hrt.2003.025270.

5. Levy D, Larson MG, Vasan RS, et al. The progression from hypertension to congestive heart failure. JAMA 275(20):1557-1562. DOI: 10.1001/ jama.1996.03530440037034.

6. Ho KKL, Pinsky JL, Kannel WB, et al. The epidemiology of heart failure: the Framingham study. J Am Coll Cardiol 1993;22(4 Suppl A):6A-13A. DOI: 10.1016/0735-1097(93)90455-a.

7. He J, Ogden LG, Bazzano LA, et al. Risk factors for congestive heart failure in US men and women: NHANES I epidemiologic follow-up study. Arch Intern Med 2001;161(7):996-1002. DOI: 10.1001/ archinte.161.7.996.

8. Owan TE, Hodge DO, Herges RM, et al. Trends in prevalence and outcome of heart failure with preserved ejection fraction. N Engl J Med 2006;355(3):251-259. DOI: 10.1056/NEJMoa052256.

9. Brouwers FP, de Boer RA, van der Harst $P$, et al. Incidence and epidemiology of new onset heart failure with preserved vs. reduced ejection fraction in a community-based cohort: 11-year follow-up of prevend. Eur Heart J 2013;34(19):1424-1431. DOI: 10.1093/eurheartj/ eht066.

10. Oldenburg O, Lamp B, Faber L, et al. Sleep-disordered breathing in patients with symptomatic heart failure a contemporary study of prevalence in and characteristics of 700 patients. Eur J Heart Fail 2007;9(3):251-257. DOI: 10.1016/j.ejheart.2006.08.003.

11. Chan J, Sanderson J, Chan W, et al. Prevalence of sleep-disordered breathing in diastolic heart failure. Chest 1997;111(6):1488-1493. DOI: 10.1378/chest.111.6.1488.

12. Sin DD, Fitzgerald F, Parker JD, et al. Risk factors for central and obstructive sleep apnea in 450 men and women with congestive heart failure. Am J Respir Crit Care Med 1999;160(4):1101-1106. DOI: 10.1164/ajrccm.160.4.9903020.

13. Shahar E, Whitney CW, Redline S, et al. Sleep-disordered breathing and cardiovascular disease: cross-sectional results of the sleep heart health study. Am J Respir Crit Care Med 2001;163(1):19-25. DOI: 10.1164/ajrccm.163.1.2001008.

14. Grimm W, Sass J, Sibai E, et al. Severe central sleep apnea is associated with atrial fibrillation in patients with left ventricular systolic dysfunction. Pacing Clin Electrophysiol 2015;38(6):706-712. DOI: 10.1111/pace.12495.

15. Efken C, Bitter T, Prib N, et al. Obstructive sleep apnoea: longer respiratory event lengths in patients with heart failure. Eur Respir J 2013;41(6):1340-1346. DOI: 10.1183/09031936.00082212.

16. Malhotra A, White DP. Obstructive sleep apnoea. Lancet 2002;360(9328):237-245. DOI: 10.1016/S0140-6736(02)09464-3.

17. Bradley TD, Floras JS. Sleep apnea and heart failure: part I: obstructive sleep apnea. Circulation 2003;107(12):1671-1678. DOI: 10.1161/01. CIR.0000061757.12581.15.

18. Parati G, Lombardi C, Hedner J, et al. Position paper on the management of patients with obstructive sleep apnea and hypertension: joint recommendations by the european society of hypertension, by the european respiratory society and by the members of european COST (cooperation in scientific and Technological research) ACTION B26 on obstructive sleep apnea. J Hypertens 2012;30(4):633-646. DOI: 10.1097/HJH.0b013e328350e53b.

19. Eckert DJ, Malhotra A, Jordan AS. Mechanisms of apnea. Prog Cardiovasc Dis 2009;51(4):313-323. DOI: 10.1016/j.pcad.2008.02.003.

20. Kohnlein T, Welte T, Tan LB, et al. Central sleep apnoea syndrome in patients with chronic heart disease: a critical review of the current 
literature. Thorax 2002;57(6):547-554. DOI: 10.1136/thorax.57. 6.547.

21. Szollosi I, Roebuck T, Thompson B, et al. Lateral sleeping position reduces severity of central sleep apnea/Cheyne-stokes respiration. Sleep 2006;29(8):1045-1051. DOI: 10.1093/sleep/29.8.1045.

22. Kasai T, Floras JS, Bradley TD. Sleep apnea and cardiovascular disease: a bidirectional relationship. Circulation 2012;126(12):1495-1510. DOI: 10.1161/CIRCULATIONAHA.111.070813.

23. Hayes $\mathrm{D}$, Anstead $\mathrm{Ml}$, Ho J, et al. Insomnia and chronic heart failure. Heart Fail Rev 2009;14(3):171-182. DOI: 10.1007/s10741-008-9102-1.

24. American Academy of Sleep Medicine Task Force. Sleep-related breathing disorders in adults: recommendations for syndrome definition and measurement techniques in clinical research. the report of an american academy of sleep medicine task force. Sleep 1999;22(5):667-689. DOI: 10.1093/sleep/22.5.667.

25. Redline S, Budhiraja R, Kapur V, et al. The scoring of respiratory events in sleep: reliability and validity. J Clin Sleep Med 2007;3(2):169-200. DOI: $10.5664 /$ jcsm.26818.

26. Yumino D, Bradley TD. Central sleep apnea and cheyne-stokes respiration. Proc Am Thorac Soc 2008;5(2):226-236. DOI: 10.1513/ pats.200708-129MG.

27. Peppard PE, Young $\mathrm{T}$, Barnet $\mathrm{JH}$, et al. Increased prevalence of sleep-disordered breathing in adults. Am J Epidemiol 2013;177(9): 1006-1014. DOI: 10.1093/aje/kws342.

28. Lévy P, Kohler M, McNicholas WT, et al. Obstructive sleep apnoea syndrome. Nat Rev Dis Prim 2015;1(1):15015. DOI: 10.1038/ nrdp.2015.15.

29. Redeker NS, Adams L, Berkowitz R, et al. Nocturia, sleep and daytime function in stable heart failure. J Card Fail 2012;18(7):569-575. DOI: 10.1016/j.cardfail.2012.05.002.

30. Arzt M, Young T, Finn L, et al. Sleepiness and sleep in patients with both systolic heart failure and obstructive sleep apnea. Arch Intern Med 2006;166(16):1716-1722. DOI: 10.1001/archinte.166.16.1716.

31. McKelvie RS, Moe GW, Cheung A, et al. The 2011 canadian cardiovascular society heart failure management guidelines update: focus on sleep apnea, renal dysfunction, mechanical circulatory support, and palliative care. Can J Cardiol 2011;27(3):319-338. DOI: 10.1016/j.cjca.2011.03.011.

32. Sateia MJ. International classification of sleep disorders-third edition. Chest 2014;146(5):1387-1394. DOI: 10.1378/chest.14-0970.

33. Farre R, Montserrat JM, Navajas D. Noninvasive monitoring of respiratory mechanics during sleep. Eur Respir J 2004;24(6): 1052-1060. DOI: 10.1183/09031936.04.00072304.
34. Fung JWH, Li TST, Choy DKL, et al. Severe obstructive sleep apnea is associated with left ventricular diastolic dysfunction. Chest 2002;121(2):422-429. DOI: 10.1378/chest.121.2.422.

35. Otto ME, Belohlavek M, Romero-Corral A, et al. Comparison of cardiac structural and functional changes in obese otherwise healthy adults with versus without obstructive sleep apnea. Am J Cardiol 2007;99(9):1298-1302. DOI: 10.1016/j.amjcard.2006.12.052.

36. Romero-Corral A, Somers VK, Pellikka PA, et al. Decreased right and left ventricular myocardial performance in obstructive sleep apnea. Chest 2007;132(6):1863-1870. DOI: 10.1378/chest.07-0966.

37. Sidana J, Aronow WS, Ravipati G, et al. Prevalence of moderate or severe left ventricular diastolic dysfunction in obese persons with obstructive sleep apnea. Cardiology 2005;104(2):107-109. DOI: $10.1159 / 000087128$.

38. Bitter T, Faber L, Hering D, et al. Sleep-disordered breathing in heart failure with normal left ventricular ejection fraction. Eur J Heart Fail 2009;11(6):602-608. DOI: 10.1093/eurjhf/hfp057.

39. Bucca CB, Brussino L, Battisti A, et al. Diuretics in obstructive sleep apnea with diastolic heart failure. Chest 2007;132(2):440-446.

40. Berry RB. Obstructive sleep apnea treatment overview and medical treatments. In: Fundamentals of Sleep Medicine 2012. 299-309.

41. Terziyski K, Draganova A. Central sleep apnea with cheynestokes breathing in heart failure - from research to clinical practice and Beyond. Adv Exp Med Biol 2018;1067:327-351. DOI: 10.1007/5584_2018_146.

42. Bradley TD, Logan AG, Kimoff RJ, et al. Continuous positive airway pressure for central sleep apnea and heart failure. NEJM 2005;353(19):2025-2033. DOI: 10.1056/NEJMoa051001.

43. Cowie MR, Woehrle H, Wegscheider K, et al. Adaptive servoventilation for central sleep apnea in systolic heart failure. $\mathrm{N}$ Engl J Med 2015;373(12):1095-1105. DOI: 10.1056/NEJMoa1506459.

44. Perger E, Lyons OD, Inami T, et al. Predictors of 1-year compliance with adaptive servoventilation in patients with heart failure and sleep disordered breathing: preliminary data from the ADVENT-HF trial. Eur Respir J 2019;53(2). DOI: 10.1183/13993003.016262018.

45. Ponikowski P, Javaheri S, Michalkiewicz D, et al. Transvenous phrenic nerve stimulation for the treatment of central sleep apnoea in heart failure. Eur Heart J 2012;33(7):889-894. DOI: 10.1093/eurheartj/ ehr298.

46. Abraham WT, Jagielski D, Oldenburg O, et al. Phrenic nerve stimulation for the treatment of central sleep apnea. JACC Hear Fail 2015;3(5):360-369. DOI: 10.1016/j.jchf.2014.12.013. 